\title{
Long-Term Follow-up of Enhanced Holter- Electrocardiography Monitoring in Acute Ischemic Stroke
}

\author{
Rolf Wachter, ${ }^{\mathrm{a}, \mathrm{b}, \mathrm{c}}$ Mark Weber-Krüger, ${ }^{\mathrm{c}}$ Gerhard F. Hamann, ${ }^{\mathrm{d}}$ Pawel Kermer, ${ }^{\mathrm{e}}$ Jan Liman, ${ }^{\mathrm{f}}$ \\ Meinhard Mende, ${ }^{\mathrm{g}}$ Joachim Seegers, ${ }^{\mathrm{h}, \mathrm{i}}$ Katrin Wasser, ${ }^{\mathrm{f}}$ Sonja Gröschel, ${ }^{\mathrm{j}}$ Timo Uphaus, ${ }^{\mathrm{j}}$ \\ Holger Poppert, ${ }^{\mathrm{k}, \mathrm{l}}$ Martin Köhrmann, ${ }^{\mathrm{m}}$ Markus Zabel, ${ }^{\mathrm{b}, \mathrm{c}}$ Ulrich Laufs, ${ }^{a}$ Peter U. Heuschmann, ${ }^{\mathrm{n}, \mathrm{o}, \mathrm{p}}$ \\ David Conen, ${ }^{\mathrm{q}}$ Klaus Gröschel, ${ }^{\mathrm{j}}$ on Behalf of the Find-AFrandomised Investigators and Coordinators \\ ${ }^{a}$ Clinic and Policlinic for Cardiology, University Hospital Leipzig, Leipzig, Germany \\ ${ }^{b}$ DZHK (German Center for Cardiovascular Research), partner site Göttingen, Göttingen, Germany \\ 'Clinic for Cardiology and Pneumology, University Medical Center Göttingen, Göttingen, Germany \\ ${ }^{d}$ Clinic for Neurology and Neurorehabilitation, Bezirkskrankenhaus Günzburg, Günzburg, Germany \\ ${ }^{e}$ Clinic for Neurology, Nordwest-Krankenhaus Sanderbusch, Sande, Germany \\ ${ }^{f}$ Clinic for Neurology, University of Göttingen, Göttingen, Germany \\ ${ }^{9}$ Center for Clinical Trials and Institute for Medical Informatics, Statistics and Epidemiology, University Leipzig, Leipzig, Germany \\ ${ }^{h}$ Division of Electrophysiology, Department of Internal Medicine II, Klinikum Landshut, Landshut, Germany \\ 'Department of Internal Medicine II, University Hospital Regensburg, Regensburg, Germany \\ jDepartment of Neurology, University Medical Centre of the Johannes Gutenberg University Mainz, Mainz, Germany \\ ${ }^{k}$ Clinic for Neurology, Helios Klinikum München-West, München, Germany \\ 'Department of Neurology, University Hospital Rechts der Isar, München, Germany \\ ${ }^{m}$ Department of Neurology, University Hospital Essen, Essen, Germany \\ nInstitute for Clinical Epidemiology and Biometry, University of Würzburg, Würzburg, Germany \\ ${ }^{\circ}$ Clinical Trial Centre, University Hospital Würzburg, Würzburg, Germany \\ ${ }^{p}$ Comprehensive Heart Failure Center, University of Würzburg, Würzburg, Germany \\ ${ }^{9}$ Population Health Research Institute, McMaster University, Hamilton, ON, Canada
}

Background and Purpose Prolonged electrocardiography (ECG)-monitoring in stroke patients improves the detection of paroxysmal atrial fibrillation (pAF). However, most randomized studies only had short follow-up. We aimed to provide 3-year follow-up data for AF detection and stroke recurrence risk.

Methods We randomized 402 patients aged $\geq 60$ years with acute ischemic strokes without AF to either enhanced and prolonged monitoring (EPM; 3×10-day Holter-ECG-monitoring) or standard-of-care ( $\geq 24$ hours ECG-monitoring). The endpoint of the current analysis was AF within 36 months analyzed by intention to treat. Long-term follow-up was performed for 36 months. Results Two hundred and seventy-four patients (80\%) participated in the extended follow-up (median duration of follow-up was 36 months [interquartile range, 12 to 36]). During the first 6 months, more AF was documented in the EPM arm compared to the control arm (13.5\% vs. $5.1 \% ; 95 \%$ confidence interval, $2.9 \%$ to $14.4 \% ; P=0.004$ ). During months 6 to 36 , AF was less detected in the EPM intervention arm than in the control arm (2.0\% vs. $7.3 \%$; $95 \%$ confidence interval, $0.7 \%$ to $9.9 \% ; P=0.028$ ). Overall, the detection rate of AF within 36 months was numerically higher within the EPM group (15.0\% vs. $11.1 \%, P=0.30)$. Numerically less patients in the EPM arm had recurrent ischemic strokes (5.5\% vs. 9.1\%, $P=0.18$ ), transient ischemic attacks
Correspondence: Rolf Wachter Clinic and Policlinic for Cardiology, University Hospital Leipzig, 04103 Leipzig, Germany

Tel: +49-341-97-12650

Fax: +49-341-97-12659

E-mail: Rolf.Wachter@medizin.unileipzig.de

https://orcid.org/0000-0003-2231-2200

Received: April 1, 2021

Revised: September 20, 2021

Accepted: September 23, 2021 
( $3.0 \%$ vs. $4.5 \%, P=0.44$ ) or died ( $4.5 \%$ vs. $6.6 \%, P=0.37$ ).

Conclusions Enhanced and prolonged ECG monitoring increased AF detection during the first six months, but there was significantly more clinical AF during months 6 to 36 observed in the usual-care arm. This suggests that EPM leads to an earlier detection of clinically relevant AF.

Keywords Stroke; Atrial fibrillation; Electrocardiographic monitoring; Randomized controlled trial

\section{Introduction}

Atrial fibrillation (AF) is a major cause of ischemic strokes. AF-related strokes are more severe than those of other etiologies $^{1}$ and stroke patients with AF exhibit a high risk of recurrent ischemic events. ${ }^{2} \mathrm{AF}$ might escape routine stroke etiology work-up as paroxysmal AF (pAF) occurs intermittently. The identification of pAF is of major clinical importance as it usually changes the secondary stroke prevention strategy with respect to the initiation of oral anticoagulation instead of antiplatelet therapy. This leads to a $40 \%$ relative risk reduction of recurrent strokes events. ${ }^{3}$

So far, three randomized trials have demonstrated that a high proportion of patients with PAF can be identified by prolonged electrocardiography (ECG)-monitoring ${ }^{4-6}$ compared to a standard stroke workup. However, the clinical relevance of AF detected by different means is unknown. For instance, short episodes detected by implantable cardiac monitors are a frequent finding in patients with cardiovascular risk factor $(29 \%$ within 18 months) while the overall stroke rate is low. ${ }^{7}$

A latent or early symptomatic stage is a key principle of a screening program. ${ }^{8}$ This principle has not yet been proven for AF detection in stroke patients. We aimed to follow-up patients after a 6-month monitoring period for additional 2.5 years to investigate if (1) detection rate of AF during follow-up is different between both study arms and (2) ischemic stroke rate is different between both study arms.

\section{Methods}

\section{Study design and patients}

Find- $\mathrm{AF}_{\text {RANDOMISED }}$ was an investigator-initiated randomized, controlled, open-label trial, conducted at four certified stroke centers in Germany. It is registered with clinicaltrials.gov (NCT01855035).

The study protocol was approved by the individual ethics boards at each study site. Patients 60 years or older with ischemic strokes and symptoms $\leq 7$ days were prospectively included after signing written and informed consent. Patients with known $A F$, another indication or a contraindication for oral an- ticoagulation were excluded. We also excluded those with symptomatic ipsilateral stenoses ( $>50 \%$, North American Symptomatic Carotid Endarterectomy Trial [NASCET] classification). The details of all inclusion and exclusion criteria have been published previously. ${ }^{6,9}$

In an amendment dated July 22nd, 2014, we extended the follow-up duration to 36 months after the index event. All patients still in the trial after 12 months were contacted in order to acquire informed consent for the extended trial phase.

\section{Randomization and masking}

Eligible patients were randomized in a 1:1 ratio to either enhanced and prolonged monitoring (EPM) by means of three 10day Holter-ECGs which were analyzed in a central core laboratory (intervention group) or standard workup, including $\geq 24$ hours of ECG-monitoring (Holter-ECG and/or telemetry). ${ }^{6}$

\section{Procedures}

Clinical follow-up visits took place $3,6,12,18,24,30$, and 36 months after the index event in both groups. Follow-up visits beyond 12 months were conducted by telephone. We collected information on recent morbidity (including a new diagnosis of AF/atrial flutter), current medication, adverse events, guideline adherence, and disability (modified Rankin Scale). If AF was reported, ECGs tracings were requested from treating physicians/ hospitals. Additional diagnostic work-up to identify pAF was not recommended.

Patients in the intervention group received a total of three 10-day two-channel (five-lead) Holter-ECG (CardioMem 3000, Getemed, Teltow, Germany) at baseline and at the 3 and 6 months follow-up visit, respectively. All ECGs in the intervention group were analyzed by an ECG core laboratory, following a predefined standard operating procedure. An alternative thumb-sensor ECG-device was offered to those who refused to repeat the Holter-ECG. The control group received $\geq 24$ hours of ECG-monitoring (telemetry and or Holter-ECG), according to local standards.

AF was defined according to the 2010 European Society of Cardiology guideline ${ }^{10}$ including only episodes detected on a 12lead ECG or that lasted at least $\geq 30$ seconds on a rhythm strip. 


\section{Outcomes}

The primary endpoint of the trial was the ECG-documentation of atrial fibrillation (AF) or atrial flutter within 6 months after randomization and before a recurrent stroke. All potential episodes were evaluated by an expert primary endpoint adjudication committee (D.C., U.L., and M.Z.), which was blinded to all other clinical data.

The extended follow-up collected outcome information until 36 months after randomization. Major outcomes included the detection of AF, the recurrence of stroke or systemic embolism, transient ischemic attack (TIA) and death. Events that potentially qualified as recurrent cerebral events (strokes or TIAs) were assessed by an independent stroke adjudication committee (M.K., H.P., and P.U.H.), masked to all data regarding the primary endpoint and to the allocated study group. Stroke was defined according to the criteria of the World Health Organization as an acute onset of focal or global neurologic deficits of presumed vascular origin lasting for more than 24 hours or resulting in death before 24 hours. In case that patients received thromboIytic or recanalization therapy, the event was classified as stroke irrespective of duration of clinical symptoms. If the clinical symptoms last less than 24 hours and the patients were not receiving thrombolytic or recanalization therapy or dying within 24 hours, the event was classified as TIA. TIAs with a corresponding lesion on brain imaging were counted as strokes.

\section{Statistical analysis}

Baseline characteristics are presented as means and standard deviation (SD) or frequencies and percentages. For variables that were not normally distributed, the median and interquartile ranges (IQR) are shown. Comparison of event rates in cross tables was performed by chi-square test. Frequency differences are estimated with 95\% confidence intervals (Cls).

Because death is a competing risk for detection of atrial fibrillation/flutter, cumulative incidences following the methods of Fine \& Gray ${ }^{11}$ were calculated, depicted and tested. Times to study end were considered as censored. To separate AF detection in the treatment period (0 to 6 months) from those of the follow-up, conditional survival methods were applied for estimation of cumulative incidence of AF after 6 months. A Cox model with time-dependent covariates was built to model the different risk for $\mathrm{AF}$ in the treatment period and afterwards. Time to stroke or death was analyzed by Kaplan-Meier method and log rank test.

Significance level was determined 5\% for two-tailed tests. All statistical analyses were performed with SPSS version 26 (IBM Co., Armonk, NY, USA) and R version 3.6 (R Foundation for Statistical Computing, Vienna, Austria).

\section{Results}

Baseline characteristics and results until 12 months of follow-up have been published and are included here to depict the full study results. ${ }^{6}$ One thousand six hundred four patients $\geq 60$ years with International Classification of Diseases (ICD) diagnosis 63.x were screened and finally 402 patients were enrolled and randomized at four study centers in Germany from May 8th, 2013 until August 31st, 2014. Four patients were randomized erroneously (two in each group) and were excluded from the final analysis. Two hundred patients were assigned to the intervention group and 198 to the control group. The study population had a mean \pm SD age of $73 \pm 7$ years (range, 60 to 96$)$. Forty percentage (160 patients) were female.

Details on the monitoring in the intervention and control group have been published previously. ${ }^{6}$ In brief, in the intervention group, 197 patients (99.5\%), 128 (75.3\% of those remaining in the study and without detected AF) and 116 patients (75.8\%) underwent study monitoring at baseline, after 3 and after 6 months, respectively.

In the control group, 188 of 198 patients (95\%) underwent Stroke-Unit telemetry for a median duration of 73 hours (IQR, 54 to 84$)$ and 149 of 198 patients (75\%) received additional Holter-ECG-monitoring for a median of 24 hours (IQR, 22 to 25). One hundred and eighty-one of 198 patients (91.4\%) in the control arm and 178 of 200 patients (89.0\%) in the intervention arm had at least 24 hours of monitoring outside the study monitoring.

The primary trial ended as planned after all patients had completed 12 months of follow-up and the last patient's final visit took place on September 16th, 2015. After 12 months, all 342 patients still in the trial were offered an extended follow-up for an additional 2 years, and 274 (80.1\%) consented for the extended follow-up. Details are shown in Figure 1 and baseline characteristics of patients consenting for extended follow-up are shown in Table 1. Patients consenting for extended follow-up were on average 3 years younger than those who did not give consent, tended to have less comorbidities, more often had a history of myocardial infarction and coronary artery disease, and had lower initial National Institutes of Health Stroke Scale (NIHSS) score (Supplementary Table 1).

\section{Detection of atrial fibrillation}

Within 6 months after randomization, newly diagnosed AF occurred in 13.5\% (27 of 200 patients) in the group assigned to EPM, as compared to $5.1 \%$ (10 of 198 patients) in the control group (absolute difference, 8.4\%; 95\% $\mathrm{Cl}, 2.9$ to $14.4 ; P=0.004$; needed to screen $n=12$ ). Details on the AF detection by the study intervention are shown in Supplementary Table 2. One 


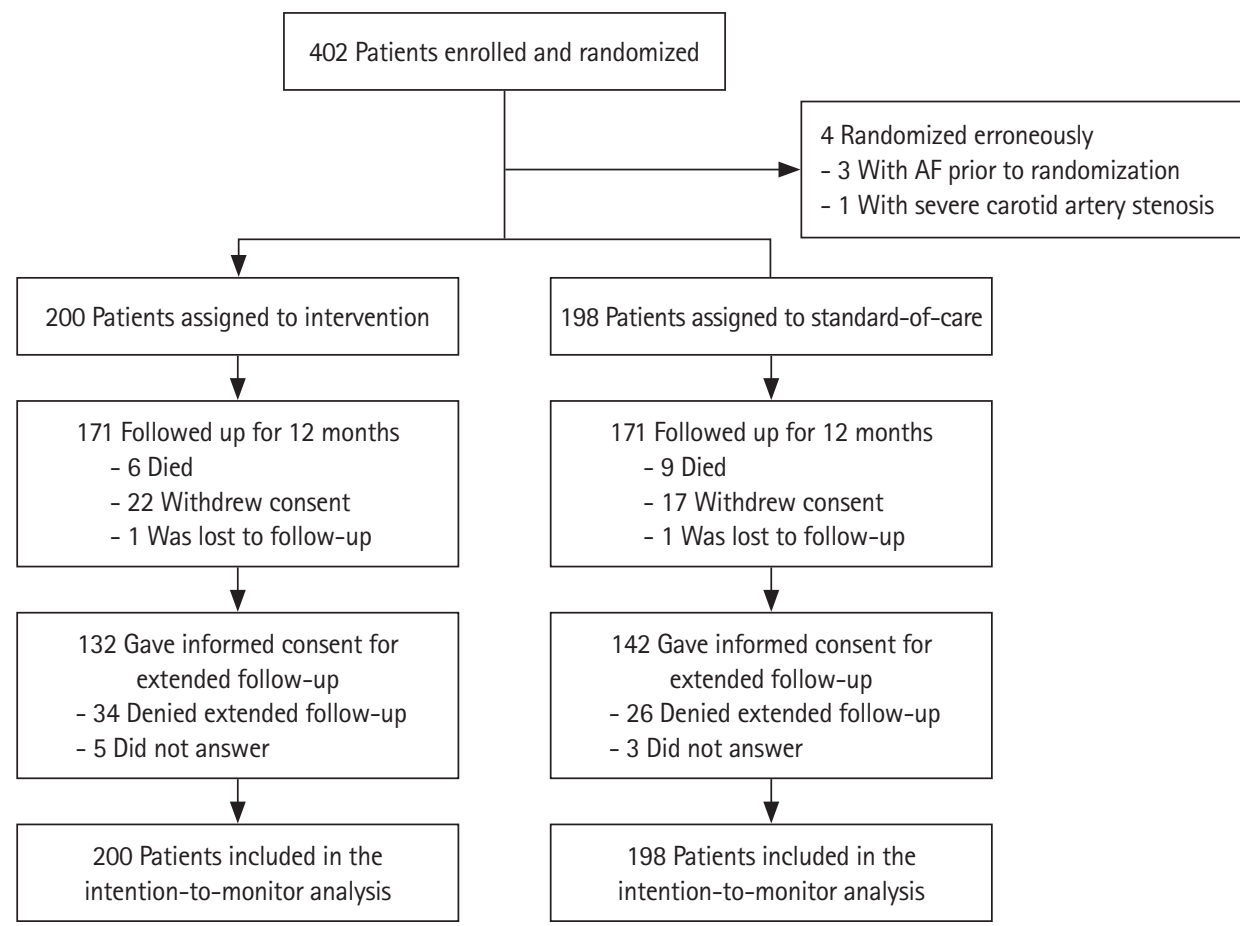

Figure 1. Trial profile. Randomization, electrocardiography monitoring, follow-up, and participation in the extended follow-up. Four hundred and two patients were enrolled and randomized, four patients were randomized erroneously and were excluded from further analysis. Two hundred and seventy-four from 342 participants gave informed consent for extended follow-up. AF, atrial fibrillation.

Table 1. Baseline characteristics of the patients participating in the extended follow-up

\begin{tabular}{|c|c|c|c|}
\hline Characteristic & Intervention group $(n=132)$ & Control group $(n=142)$ & $P$ \\
\hline Age (yr) & $70.5 \pm 6.8$ & $72.3 \pm 7.4$ & 0.03 \\
\hline Female sex & $51(38.6)$ & 48 (33.8) & 0.45 \\
\hline \multicolumn{4}{|l|}{ Medical history } \\
\hline Hypertension & $101(76.5)$ & 112 (78.9) & 0.67 \\
\hline Diabetes & $29(22.0)$ & $35(24.6)$ & 0.67 \\
\hline Hyperlipidemia & $46(34.8)$ & $64(45.1)$ & 0.11 \\
\hline \multicolumn{4}{|l|}{ Smoking status } \\
\hline Current smoker & $21(15.9)$ & $24(16.9)$ & 0.87 \\
\hline Previous smoker & $41(31.1)$ & $42(29.6)$ & 0.79 \\
\hline Previous ischemic stroke & $20(15.1)$ & $28(19.7)$ & 0.34 \\
\hline Previous transient ischemic attack & $11(8.3)$ & $14(9.6)$ & 0.68 \\
\hline Heart failure & $5(3.8)$ & $8(5.6)$ & 0.58 \\
\hline Myocardial infarction & $14(10.6)$ & $12(8.5)$ & 0.68 \\
\hline Coronary artery disease & $18(13.6)$ & $26(18.3)$ & 0.33 \\
\hline Mean creatinine $(\mathrm{mg} / \mathrm{dL})^{*}$ & $1.0 \pm 0.4$ & $1.0 \pm 0.3$ & 0.39 \\
\hline Mean ejection fraction $(\%)^{+}$ & $61 \pm 8$ & $60 \pm 9$ & 0.85 \\
\hline Symptoms $>24$ hours & $123(93.2)$ & $134(94.4)$ & 0.80 \\
\hline Symptoms $<24$ hours and DWI lesion in MRI & $9(6.8)$ & $8(5.6)$ & 0.80 \\
\hline Lacunar lesion on brain imaging & 42/96 (43.8) & 46/94 (48.9) & 0.56 \\
\hline TOAST classification & & & 0.02 \\
\hline Large artery atherosclerosis & $3(2.3)$ & $1(0.7)$ & \\
\hline Cardioembolism & $37(28.0)$ & 19 (13.4) & \\
\hline
\end{tabular}


Table 1. Continued

\begin{tabular}{|c|c|c|c|}
\hline Characteristic & Intervention group $(n=132)$ & Control group $(n=142)$ & $P$ \\
\hline Small-vessel occlusion & $40(30.3)$ & $50(35.2)$ & \\
\hline Stroke of other determined etiology & $0(0)$ & $1(0.7)$ & \\
\hline Stroke of undetermined etiology & $52(39.4)$ & $71(50.0)$ & \\
\hline Score on NIHSS ${ }^{*}$ & $3(1-4)$ & $2(1-4)$ & 0.11 \\
\hline Time from symptom onset to randomization (day) & $3(2-4)$ & $3(2-5)$ & 0.34 \\
\hline Mean $\mathrm{CHA}_{2} \mathrm{DS}_{2}$ VASc score & $4.5 \pm 1.3$ & $4.7 \pm 1.2$ & 0.48 \\
\hline $\mathrm{CHA}_{2} \mathrm{DS}_{2}$ VASc score & & & 0.32 \\
\hline 2 & $10(7.6)$ & $7(4.9)$ & \\
\hline 3 & $21(15.9)$ & $13(9.2)$ & \\
\hline 4 & $32(24.2)$ & $46(32.4)$ & \\
\hline 5 & $41(31.1)$ & $41(28.9)$ & \\
\hline 6 & $24(18.2)$ & $26(18.3)$ & \\
\hline 7 & $4(3.0)$ & $8(5.6)$ & \\
\hline 8 & $0(0)$ & $1(0.7)$ & \\
\hline Mean $\mathrm{CHADS}_{2}$ score" & $3.3 \pm 0.9$ & $3.5 \pm 0.9$ & 0.89 \\
\hline $\mathrm{CHADS}_{2}$ score & & & 0.07 \\
\hline 2 & $27(20.5)$ & 16 (11.3) & \\
\hline 3 & $53(40.2)$ & 65 (45.8) & \\
\hline 4 & 42 (32.6) & 41 (28.9) & \\
\hline 5 & $9(6.8)$ & $20(14.1)$ & \\
\hline 6 & $1(0.8)$ & $0(0)$ & \\
\hline 4-6 pooled & $52(39.4)$ & 61 (43.0) & \\
\hline
\end{tabular}

Values are presented as mean \pm standard deviation, number (\%), or median (interquartile range).

DWI, diffusion-weighted imaging; MRI, magnetic resonance imaging; TOAST, Trial of Org 10172 in Acute Stroke Treatment; NIHSS, National Institutes of Health Stroke Scale.

${ }^{*}$ Data were missing for one patient in the intervention group; ${ }^{\dagger}$ Data were missing in 22 patients in the intervention group and 20 patients in the control group; ${ }^{7}$ Scores on the National Institutes of Health $(\mathrm{NIH})$ Stroke Scale range from 0 to 42, higher scores indicate a greater neurological deficit. Data were missing for one patient in the control group; ${ }^{5}$ Scores on the $\mathrm{CHA}_{2} \mathrm{DS}_{2} \mathrm{VASc}$ risk assessment range from 0 to 9 , with higher scores indicating a greater risk of thromboembolic events; "Scores on the $\mathrm{CHADS}_{2}$ risk assessment range from 0 to 6 , with higher scores indicating a greater risk of thromboembolic events.

case of $\mathrm{AF}$ in the control arm was initially not reported by the study site and not included in the primary publication, ${ }^{6}$ but was identified during final study close out and was positively adjudicated by the endpoint committee. From the end of the study intervention (6 months) to the end of the follow-up (36 months), there were significantly more new AF cases detected in the control arm than in the intervention arm (12/171 [7.0\%] vs. $3 / 154$ [1.9\%], absolute difference, $5.1 \% ; 95 \% \mathrm{Cl}, 0.6 \%$ to $10.1 \%$ ) (Supplementary Figure 1). The hazard ratio (HR) is 0.33 $(95 \% \mathrm{Cl}, 0.16$ to $0.68 ; P=0.003)$ for the estimated AF risk in the control arm up to 6 months calculated with an age-adjusted Cox regression model. In the time period of 6 to 36 month the $\mathrm{HR}$ is 10.2 (95\% Cl, 2.36 to $43.7 ; P=0.002)$.

Two thirds of AF cases during extended follow-up were detected by 12-channel surface ECG. Table 2 shows details on AF detection during extended follow-up. The difference in AF incidence between the two arms diminished over time. After 36 months, the cumulative incidence of the $\mathrm{AF}$ detection rate was $15.9 \%$ in the EPM arm and $12.8 \%$ in the control arm $(P=0.17)$ (Figure 2). Of note, 25 of the total $30 \mathrm{AF}$ cases (83\%) in the intervention arm were detected by the study intervention. Of all 52 patients, in which AF was detected during follow-up, 18 $(34.6 \%)$ of the index strokes were documented as cardioembolic, $11(21.2 \%)$ as small vessel occlusion and 19 were classified as stroke of unknown cause (36.5\%).

\section{Anticoagulation for atrial fibrillation}

Oral anticoagulation was started in all 52 patients with detected $\mathrm{AF}$ and the anticoagulation rate was $97 \%$ after 12 months, 97\% after 18 months, 94\% after 24 months, and 100\% after 30 and 36 months.

\section{Stroke, transient ischemic attack, and death}

During the whole study period, there were 13 stroke events in 
Table 2. Endpoints within 3 years of follow-up

\begin{tabular}{lcccc}
\hline & EPM $(\%)(n=200)$ & Usual care $(\%)(n=198)$ & HR $(95 \% \mathrm{Cl})$ & $P$ \\
\hline Atrial fibrillation & 15.0 & 11.1 & $1.45(0.83-2.51)$ & 0.187 \\
TIA & 3.0 & 4.5 & $0.65(0.22-1.98)$ & 0.448 \\
Stroke & 5.5 & 9.1 & $0.61(0.29-1.29)$ & 0.191 \\
Death & 4.5 & 6.6 & $0.71(0.30-1.65)$ & 0.419 \\
Composite TIA/stroke/death & 12.5 & 19.2 & $0.65(0.39-1.07)$ & 0.090 \\
\hline
\end{tabular}

EPM, enhanced and prolonged monitoring; $\mathrm{HR}$, hazard ratio; $\mathrm{Cl}$, confidence interval; $\mathrm{TIA}$, transient ischemic attack.

Cumulative incidences of AF detection and death

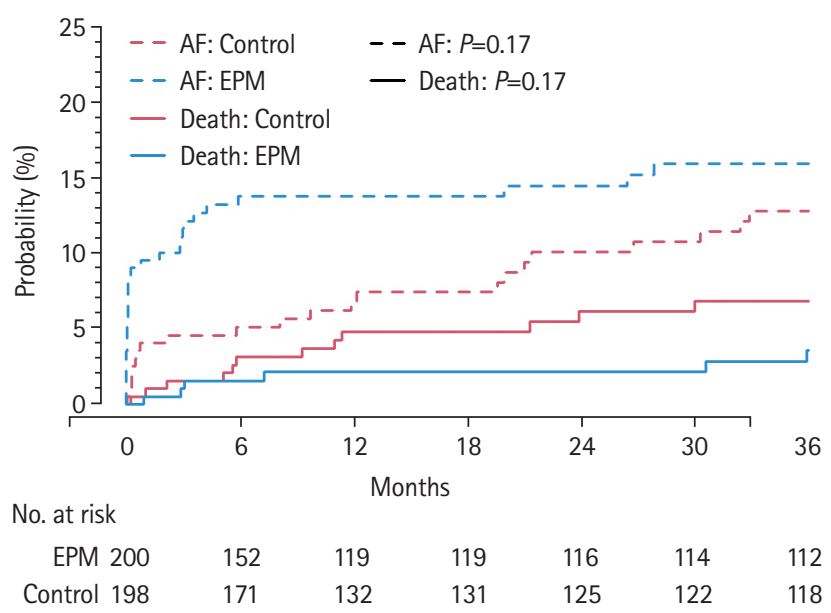

Figure 2. Cumulative incidences of atrial fibrillation (AF) detection in the presence of the competing risk death. EPM, enhanced and prolonged monitoring.

Incidence of stroke or death

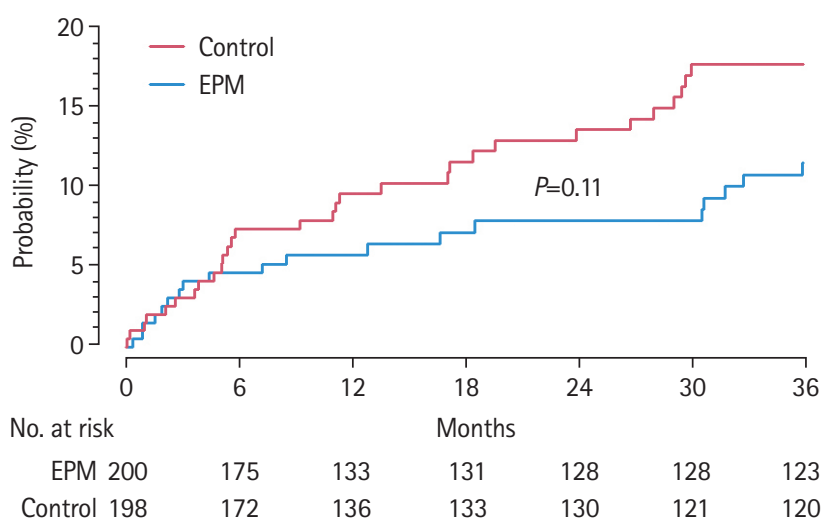

Figure 3. Cumulative incidences of the combined endpoint of stroke and death (whatever occurs first). EPM, enhanced and prolonged monitoring.

11 patients in the intervention arm (12 ischemic strokes, one hemorrhagic stroke) and 21 stroke events in 18 patients in the control arm (20 ischemic strokes, one hemorrhagic stroke). Event rates after 3 years were estimated 5.5\% versus 9.1\%
Incidence of TIA, stroke or death

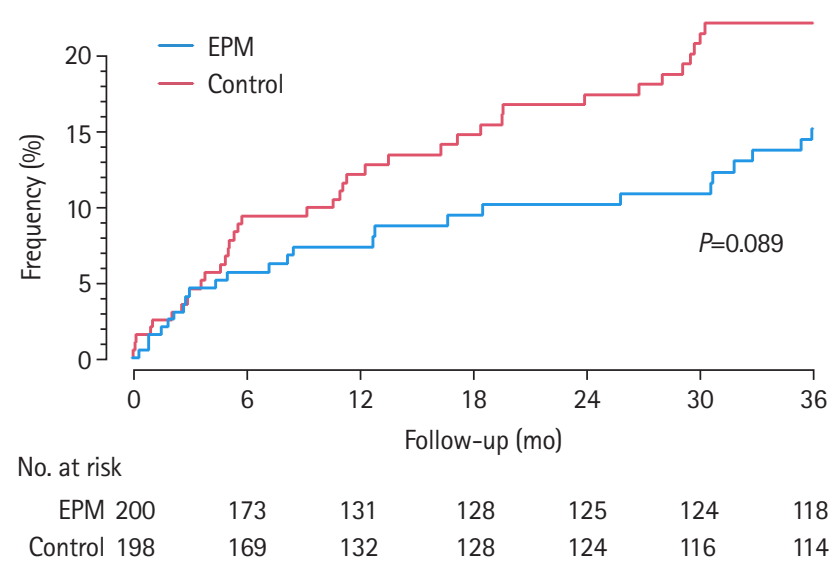

Figure 4. Cumulative incidences of the combined endpoint of transient ischemic attack (TIA), stroke, and death (whatever occurs first). EPM, enhanced and prolonged monitoring.

(odds ratio [OR], $0.58 ; 95 \% \mathrm{Cl}, 0.79$ to $1.27 ; P=0.18$ ). Both patients with hemorrhagic stroke also had an ischemic stroke event. Six patients in the intervention arm and nine patients in the control arm had a TIA (one patient in the control arm had two TIAs). No cases of systemic embolism occurred in either group. Nine patients in the intervention arm and 13 patients in the control arm died $(P=0.37)$. Since AF detection and death are competing risks, cumulative incidences were calculated and depicted (Figure 2). Figure 3 shows the incidences of the combined endpoint of stroke and death (whatever occurs first: $\mathrm{OR}, 0.63 ; 95 \% \mathrm{Cl}, 0.32$ to $1.20 ; P=0.13$ ) and Figure 4 shows the incidences of the combined endpoint of TIA, stroke, and death (whatever occurs first).

\section{Patients with atrial fibrillation and stroke or TIA}

Five patients had detection of $\mathrm{AF}$ and a recurrent ischemic cerebral event during follow-up. Sixty percentage of AF detections ( 3 out of 5 ) occurred in proximity to the recurrent ischemic cerebral event: In one patient in the intervention arm, the detection of AF was 3 days after recurrent ischemic stroke and in two patients in the control arm, the detection of AF was on 
the day of a TIA event. In the two other cases, a recurrent ischemic stroke occurred $>1$ year after the detection of AF. One patient in the intervention arm was on subtherapeutic dose of apixaban and temporally discontinued anticoagulation for pacemaker implantation when the stroke occurred and in the other patient (control arm), the stroke was associated with an ipsilateral severe carotid artery stenosis while being adequately anticoagulated with phenprocoumon (international normalized ratio [INR] on hospital admission 2.3).

\section{Discussion}

We present data on 3 years of follow-up in a study randomizing patients aged $\geq 60$ years with acute ischemic strokes to enhanced and prolonged ECG-monitoring by means of three 10day Holter-ECGs (EPM) or standard-of care to detect pAF. While there was a significant difference in the primary endpoint of first detection of atrial fibrillation prior to recurrent stroke after 6 and 12 months in the EPM group, this difference diminished over time due to a higher detection rate of $A F$ in the control group and became non-significant beyond 2 years of follow-up. We found a high adherence to anticoagulation in patients with detected new-onset AF. Importantly, there were numerically less strokes, TIAs, and deaths in the EPM arm compared to the control arm.

To date, three large randomized trials for intensified AF detection have been performed in ischemic stroke patients. The 0-Day Cardiac Event Monitor Belt for Recording Atrial Fibrillation After a Cerebral Ischemic Event (EMBRACE) trial ${ }^{4}$ applied a 30-day external event recorder in 572 patients with cryptogenic stroke, starting 75 days after the stroke event, while the Study of Continuous Cardiac Monitoring to Assess Atrial Fibrillation After Cryptogenic Stroke (CRYSTAL-AF) study ${ }^{5}$ used an implantable cardiac monitor in 441 patients with cryptogenic stroke starting 38 days after the stroke event. The third trial is the Find-AF trial, ${ }_{1}^{6}$ which also included approximately $50 \%$ of non-cryptogenic stroke patients and initiated the intervention within a median of 3 days after the qualifying ischemic stroke. All three trials found significantly more cases of $\mathrm{AF}$ in the intervention as compared to the control arm. However, the follow-up in EMBRACE was only 90 days and no information on ischemic cerebral events beyond 90 days of follow-up has been reported so far. In CRYSTAL-AF, the minimum duration of follow-up was 12 months, but only a minority of patients was followed beyond 2 years. There were numerically less strokes and TIAs in the intervention arm, but only the combined number of strokes and TIAs (20 vs. 24) has been reported and these events were not blindly adjudicated. ${ }^{12}$
Although the intervention only lasted 6 months in our study, EPM still remained superior to standard-of-care in detecting AF after 12 months ( $13.5 \%$ vs. $6.6 \%, P=0.021)$, yet losing significance after 24 months ( $14.0 \%$ vs. 9.1\%, $P=0.13)$ and 36 months (14.5\% vs. $11.1 \%, P=0.37$ ). While more patients with AF were detected in the intervention arm in the first 6 months after randomization, there were more AF cases detected in the standard-of-care arm during the following 2.5 years. This suggests that EPM mostly identified AF cases that otherwise would have been detected as clinical AF at a later stage. This is supported by the fact that most AF cases during extended follow-up were diagnosed by routine surface ECG, mostly done by the patients' general practitioner or after having had a recurrent cerebrovascular event. Interestingly, EPM within the first 6 months found as much as $5 / 6$ of all AF cases that were detected during the complete 3 years of follow-up. Our data suggest that AF episodes during a 10-day ECG monitoring are precursors of clinically relevant AF. This might be in contrast to AF episodes $>2$ minutes detected during continuous monitoring in CRYSTAL-AF: AF was diagnosed in 30\% of patients during 36 months of follow-up with an implantable loop recorder, but the detection rate in the control arm was as low as 3\% after 3 years. ${ }^{12}$ While these differences may also partly be explained by different stroke characteristics (younger patients, lower NIHSS score), the minimum duration of AF that is of clinical relevance may depend on the duration and type of monitoring. We recently showed that AF episodes of 30 seconds or longer (detected by 7-day Holter-ECG) are associated with more severe strokes (comparable to strokes in patients with $\mathrm{AF}$ on surface $E C G)$, while episodes lasting $<30$ seconds were associated with less severe strokes. ${ }^{13}$ This is an argument that the threshold of 30 seconds duration of AF on a rhythm strip seems to be adequate for Holter monitoring $\leq 10$ days. Interestingly, in patients with continuous monitoring by implanted pacemakers or defibrillators, but without prior stroke, it has recently been shown that mainly AF episodes that last for $>24$ hours are associated with a high stroke risk. ${ }^{14}$

The adherence to anticoagulation in monitoring trials seems to be higher than in randomized anticoagulation trials, e.g., the discontinuation rate in Rivaroxaban Versus Aspirin in Secondary Prevention of Stroke and Prevention of Systemic Embolism in Patients With Recent Embolic Stroke of Undetermined Source (NAVIGATE-ESUS) ${ }^{15}$ and Dabigatran Etexilate for Secondary Stroke Prevention in Patients With Embolic Stroke of Undetermined Source (RE-SPECT ESUS) ${ }^{16}$ was 15\% and 25\%, respectively. The fact that anticoagulation therapy was mandated by a specific diagnosis ( $\mathrm{AF}$ in this case) may explain the higher adherence rate in CRYSTAL-AF and Find-AF RANDOMISED. 
Although our data imply that short episodes of AF detected during 10-day Holter-ECG are of clinical relevance, this does not prove that they benefit from anticoagulation. Unfortunately, patients with short episodes of AF detected early after stroke have not been included in randomized anticoagulation trials. The question whether prolonged ECG-monitoring for AF after stroke not only increases the AF detection rate, but reduces the risk of recurrent stroke remains unknown and our trial was not powered to provide a definite answer. However, we previously reported numerically fewer stroke, TIAs, and deaths after 12 months in the EPM group ${ }^{6}$ and this trend corroborated after 36 months. This is in line with a recent meta-analysis of randomized and observational studies that found a significant reduction of cerebral ischemic events by intensified ECG monitoring. ${ }^{17}$ In summary, these findings would support the current clinical practice of treating patients with (short) AF-episodes detected after stroke with anticoagulation. Nevertheless, an adequately powered outcome trial with ischemic stroke and systemic embolism as the primary endpoint is needed to establish prolonged monitoring as an efficacious strategy for secondary stroke prevention. At least one trial, called Find-AF 2 (NCT 04371055) is underway and has already randomised more than 1,100 patients.

Find-AF $\mathrm{F}_{\text {RANDOMISED }}$ should be interpreted in the context of its limitations. The trial was conducted in Germany and we cannot rule out that results would have differed in other countries, health systems or ethnicities. Only 68\% and 65\% of patients accepted the second and third Holter-ECG. This problem may be solved by more comfortable devices, such as adhesive ECG-patches and/or by an early risk stratification based on age $_{1}{ }^{18}$ left atrial size and function, ${ }_{1}^{19}$ supraventricular ectopy, ${ }^{20}$ brain natriuretic peptides, ${ }_{1}^{21,22}$ or others as recommended in a recent consensus paper. ${ }^{23}$

The decision to extent the follow-up was made during the study conduct and only $80 \%$ of patients consented, which is a potential bias. Differences between groups in number of visits to the general practitioner, access to care, socioeconomic status could have resulted in disparities in reporting AF-related symptoms and subsequently receiving an ECG. We tried to address this potential bias by competing risk analysis, but cannot totally excluded it. Moreover, we did not specifically assess the routine monitoring that patients received during extended follow-up. According to registry data from Germany, ${ }^{24}$ most patients are treated by their General Practitioner and do not frequently receive prolonged Holter-ECG monitoring. We suppose that our study patients were treated similarly which is in line with 2/3 of AF cases during extended follow-up being detected by 12 -lead ECG, and only a minority by implantable loop re- corder or prolonged Holter-ECG monitoring.

It was not possible to blind physicians and patients to study assignment. To overcome potential bias, we chose to assess the primary endpoint "detection of AF" and the major secondary endpoint "recurrent cerebral ischemic event" by blinded endpoint committees (prospective randomized open blinded endpoint [PROBE] design). In addition, the sample size and the number of stroke events of this trial were too small to give more than a trend for recurrent cerebral ischemic events. Finally, our results were obtained in stroke patients $\geq 60$ years and $\mathrm{AF}$ prevalence is likely to be lower in younger patients. ${ }^{18}$

\section{Conclusions}

In conclusion, the extended follow-up of Find-AF $F_{\text {RANDOMISED }}$ showed that enhanced and prolonged ECG-monitoring detected AF episodes that otherwise would have likely been detected later by clinical practice. The compliance with anticoagulation in $\mathrm{AF}$ patients was excellent (>90\%) and there was a favorable trend towards fewer strokes, TIAs, and deaths in the EPM arm which might be the result of an earlier initiation of anticoagulation after AF detection. These findings provide the rationale for a larger randomized trial to prove that recurrent or continuous ECG-monitoring for AF and consecutive anticoagulation can reduce recurrent ischemic strokes.

\section{Supplementary materials}

Supplementary materials related to this article can be found online at https://doi.org/10.5853/jos.2021.01207.

\section{Disclosure}

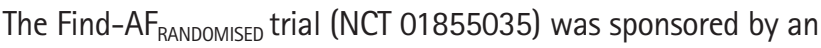
unrestricted research grant by Boehringer Ingelheim to the University of Göttingen, Germany.

Rolf Wachter reports having been an investigator or consultant for, or received fees from Bayer, Berlin Chemie, Bristol-Myers-Squibb, Boehringer Ingelheim, Boston Scientific, CVRx, Daiichi, Gilead, Johnson \& Johnson, Medtronic, Novartis, Pfizer, Sanofi, Servier outside the submitted work. He received research grants from Boehringer Ingelheim, Deutsche Forschungsgemeinschaft, European Union and Bundesministerium für Bildung und Forschung (BMBF).

Mark Weber-Krüger report grants from Boehringer Ingelheim, during the conduct of the study.

Gerhard F. Hamann reports no potential conflicts of interest.

Pawel Kermer reports grant and personal fees from Boeh- 
ringer Ingelheim, personal fees from Bayer, personal fees from Bristol-Myers Squibb outside the submitted work.

Jan Liman reports personal fees from Pfizer, Bayer Healthcare and Daiichi Sankyo outside the submitted work and grants from Boehringer Ingelheim.

Meinhard Mende reports no potential conflicts of interest.

Joachim Seegers received personal fees from Bristol-Myers Squibb outside the submitted work.

Katrin Wasser received travel compensation from Bayer.

Sonja Gröschel received travel compensation from Boehringer Ingelheim.

Timo Uphaus honoraria from Merck Serono, personal fees from Pfizer, grants from Else Kröner-Fresenius-Stiftung, outside the submitted work, outside the submitted work.

Holger Poppert reports personal fees from Bayer Healthcare, Bristol-Meyers Squipp, Daiichi Sankyo, and Pfizer outside the submitted work.

Martin Köhrmann has received honoraria and travel grants from Medtronic, BMS/Pfizer, Boehringer Ingelheim, Bayer, Novartis, Biogen, Cerevast. He received a research grant for a different study on AF detection from Boehringer Ingelheim.

Markus Zabel reports minor travel grants from Biotronik, outside the submitted work.

Ulrich Laufs reports fees for lectures from ABDA, Amgen, Ariba, AstraZeneca, Bayer, Berlin-Chemie, Boehringer, DACH, Daiichi-Sankyo, MSD, Novartis, NovoNordisk, Pfizer, Roche, Sanofi, Servier, Synlab.

Peter U. Heuschmann reports grants from University Göttin-

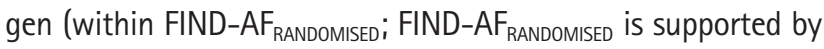
an unrestricted research grant to the University Göttingen from Boehringer-Ingelheim), during the conduct of the study; grants from German Ministry of Research and Education, German Research Foundation, European Union, Federal Joint Committee (G-BA) within the Innovationfond, Charité-Universitätsmedizin Berlin, Berlin Chamber of Physicians, German Parkinson Society, University Hospital Würzburg, Robert Koch Institute, German Heart Foundation, grants from Charité-Universitätsmedizin Berlin (within Mondafis; Mondafis is supported by an unrestricted research grant to the Charite from Bayer), grants from University Hospital Heidelberg (within RASUNOA-prime; RASUNOA-prime is supported by an unrestricted research grant to the University Hospital Heidelberg from Bayer, BMS, Boehringer-Ingelheim, Daiichi Sankyo), outside the submitted work.

David Conen has received consultant or speaker fees from Servier Canada.

Klaus Gröschel reports personal fees and non-financial support from Bayer, personal fees and non-financial support and grant from Boehringer Ingelheim, personal fees from Bris-
tol-Meyers Squipp, personal fees from Daiichi Sankyo, personal fees and non-financial support from Pfizer outside the submitted work.

\section{Acknowledgments}

Find- $\mathrm{AF}_{\text {RANDOMISED }}$ was funded by an unrestricted research grant from Boehringer Ingelheim, Ingelheim, Germany, to the University of Göttingen, Germany. All authors guarantee the validity, accuracy and integrity of the published data.

David Conen holds a McMaster University Department of Medicine Mid-Career Research Award. His work is supported by the Hamilton Health Sciences RFA Strategic Initiative Program.

We thank Prof. Götz Gelbrich, PhD, Würzburg, for fruitful biometrical discussions. Contributing site Investigators are listed in the Appendix.

\section{References}

1. Tu HT, Campbell BC, Christensen S, Desmond PM, De Silva DA, Parsons MW, et al. Worse stroke outcome in atrial fibrillation is explained by more severe hypoperfusion, infarct growth, and hemorrhagic transformation. Int J Stroke 2015; 10:534-540.

2. Kolominsky-Rabas PL, Weber M, Gefeller $\mathrm{O}$, Neundoerfer B, Heuschmann PU. Epidemiology of ischemic stroke subtypes according to TOAST criteria: incidence, recurrence, and longterm survival in ischemic stroke subtypes: a population-based study. Stroke 2001;32:2735-2740.

3. Freedman B, Potpara TS, Lip GY. Stroke prevention in atrial fibrillation. Lancet 2016;388:806-817.

4. Gladstone DJ, Spring M, Dorian P, Panzov V, Thorpe KE, Hall $J$, et al. Atrial fibrillation in patients with cryptogenic stroke. N Engl J Med 2014;370:2467-2477.

5. Sanna T, Diener HC, Passman RS, Di Lazzaro V, Bernstein RA, Morillo $C A$, et al. Cryptogenic stroke and underlying atrial fibrillation. N Engl J Med 2014;370:2478-2486.

6. Wachter R, Gröschel K, Gelbrich G, Hamann GF, Kermer P, Liman $\mathrm{J}$, et al. Holter-electrocardiogram-monitoring in patients with acute ischaemic stroke (Find-AF RANDOMISED $_{\text {): }}$ an open-label randomised controlled trial. Lancet Neurol 2017;16:282-290.

7. Reiffel JA, Verma A, Kowey PR, Halperin JL, Gersh BJ, Wachter $R$, et al. Incidence of previously undiagnosed atrial fibrillation using insertable cardiac monitors in a high-risk population: the REVEAL AF Study. JAMA Cardiol 2017;2:11201127.

8. Wilson JMG, Jungner G. Principles and Practice of Screening 
for Disease. Geneva, CH: World Health Organization, 1968.

9. Weber-Krüger M, Gelbrich G, Stahrenberg R, Liman J, Kermer $P$, Hamann GF, et al. Finding atrial fibrillation in stroke patients: randomized evaluation of enhanced and prolonged Holter monitoring: Find-AF(RANDOMISED). Rationale and design. Am Heart J 2014;168:438-445.

10. European Heart Rhythm Association; European Association for Cardio-Thoracic Surgery, Camm AJ, Kirchhof P, Lip GY, Schotten $U$, et al. Guidelines for the management of atrial fibrillation: the task force for the management of atrial fibrillation of the European Society of Cardiology (ESC). Eur Heart J 2010;31:2369-2429.

11. Gray RJ. A class of K-sample tests for comparing the cumulative incidence of a competing risk. Ann Stat 1988;16:11411154.

12. Brachmann J, Morillo CA, Sanna T, Di Lazzaro V, Diener HC, Bernstein RA, et al. Uncovering atrial fibrillation beyond shortterm monitoring in cryptogenic stroke patients: three-year results from the cryptogenic stroke and underlying atrial fibrillation trial. Circ Arrhythm Electrophysiol 2016;9:e003333.

13. Weber-Krüger M, Lutz C, Zapf A, Stahrenberg R, Seegers J, Witzenhausen J, et al. Relevance of supraventricular runs detected after cerebral ischemia. Neurology 2017;89:15451552.

14. Van Gelder IC, Healey JS, Crijns H, Wang J, Hohnloser SH, Gold MR, et al. Duration of device-detected subclinical atrial fibrillation and occurrence of stroke in ASSERT. Eur Heart $J$ 2017;38:1339-1344.

15. Hart RG, Sharma M, Mundl H, Kasner SE, Bangdiwala SI, Berkowitz SD, et al. Rivaroxaban for stroke prevention after embolic stroke of undetermined source. N Engl J Med 2018; 378:2191-2201.

16. Diener $H C$, Sacco RL, Easton JD, Granger $C B$, Bernstein RA, Uchiyama $S$, et al. Dabigatran for prevention of stroke after embolic stroke of undetermined source. N Engl J Med 2019;
380:1906-1917.

17. Tsivgoulis G, Katsanos AH, Grory BM, Köhrmann M, Ricci BA, Tsioufis $K$, et al. Prolonged cardiac rhythm monitoring and secondary stroke prevention in patients with cryptogenic cerebral ischemia. Stroke 2019;50:2175-2180.

18. Wachter R, Weber-Krüger M, Seegers J, Edelmann F, Wohlfahrt J, Wasser K, et al. Age-dependent yield of screening for undetected atrial fibrillation in stroke patients: the Find-AF study. J Neuro/ 2013;260:2042-2045.

19. Stahrenberg R, Edelmann F, Haase B, Lahno R, Seegers J, Weber-Krüger $M$, et al. Transthoracic echocardiography to rule out paroxysmal atrial fibrillation as a cause of stroke or transient ischemic attack. Stroke 2011;42:3643-3645.

20. Weber-Krüger $M$, Gröschel $K$, Mende $M$, Seegers J, Lahno R, Haase $B$, et al. Excessive supraventricular ectopic activity is indicative of paroxysmal atrial fibrillation in patients with cerebral ischemia. PLoS One 2013;8:e67602.

21. Wachter $R$, Lahno $R$, Haase $B$, Weber-Krüger $M$, Seegers J, Edelmann $F$, et al. Natriuretic peptides for the detection of paroxysmal atrial fibrillation in patients with cerebral ischemia: the Find-AF study. PLoS One 2012;7:e34351.

22. Wasser $K$, Weber-Krüger $M$, Gröschel $S$, Uphaus T, Liman J, Hamann GF, et al. Brain natriuretic peptide and discovery of atrial fibrillation after stroke: a subanalysis of the Find-AF $F_{\text {RAN- }}$ DOMISED Trial. Stroke 2020;51:395-401.

23. Haeusler KG, Gröschel K, Köhrmann M, Anker SD, Brachmann J, Böhm M, et al. Expert opinion paper on atrial fibrillation detection after ischemic stroke. Clin Res Cardiol 2018;107:871880.

24. Haeusler KG, Gerth A, Limbourg T, Tebbe U, Oeff M, Wegscheider $\mathrm{K}$, et al. Use of vitamin $\mathrm{K}$ antagonists for secondary stroke prevention depends on the treating healthcare provider in Germany: results from the German AFNET registry. BMC Neurol 2015;15:129. 
Supplementary Table 1. Clinical characteristics of patients giving consent to extended follow-up versus not giving consent

\begin{tabular}{|c|c|c|c|}
\hline Characteristic & Giving consent for follow-up ( $n=274)$ & Not giving consent $(n=60)$ & $P$ \\
\hline Age (yr) & $71.4 \pm 7.2$ & $74.5 \pm 8.1$ & 0.004 \\
\hline Female sex & $99(36.1)$ & $28(46.7)$ & 0.128 \\
\hline \multicolumn{4}{|l|}{ Medical history } \\
\hline Hypertension & $213(77.7)$ & 49 (81.7) & 0.108 \\
\hline Diabetes & $64(23.4)$ & $21(35.0)$ & 0.134 \\
\hline Hyperlipidemia & $110(40.1)$ & $24(40.0)$ & 0.970 \\
\hline \multicolumn{4}{|l|}{ Smoking status } \\
\hline Current smoker & $45(16.4)$ & $15(25.0)$ & 0.117 \\
\hline Previous smoker & $83(30.3)$ & $15(25.0)$ & 0.414 \\
\hline Previous ischemic stroke & $48(17.5)$ & $13(21.7)$ & 0.567 \\
\hline Previous transient ischemic attack & $25(9.1)$ & $3(5.0)$ & 0.054 \\
\hline Heart failure & $13(4.7)$ & $3(5.0)$ & 0.428 \\
\hline Myocardial infarction & $26(9.5)$ & $3(5.0)$ & 0.044 \\
\hline Coronary artery disease & $44(16.1)$ & $5(8.3)$ & 0.005 \\
\hline Mean creatinine (mg/dL) & $1.0 \pm 0.3$ & $1.1 \pm 0.5$ & 0.297 \\
\hline Mean ejection fraction $(\%)^{*}$ & $60 \pm 9$ & $60 \pm 9$ & 0.581 \\
\hline Initial score on NIHSS ${ }^{+}$ & $2(1-4)$ & $4(2-7)$ & 0.001 \\
\hline
\end{tabular}

Values are presented as mean \pm standard deviation, number $(\%)$, or median (interquartile range).

NIHSS, National Institutes of Health Stroke Scale.

${ }^{*}$ Data were missing in 54 patients in the group giving informed consent and 17 patients in the group not giving informed consent; ${ }^{\dagger}$ Scores on the National Institutes of Health $(\mathrm{NIH})$ Stroke Scale range from 0 to 42, higher scores indicate a greater neurological deficit. Data were missing for one patient in the control group. 
Supplementary Table 2. Details of patients with AF detection by the study intervention

\begin{tabular}{|c|c|c|c|c|c|}
\hline Age (yr) & Sex & Days after randomization & Holter ECG (n) & No. of AF episodes & Duration of longest episode (min) \\
\hline 75 & $\mathrm{~F}$ & 1 & 1 & 1 & 5,070 \\
\hline 76 & $\mathrm{~F}$ & 1 & 1 & 1 & 8,940 \\
\hline 85 & $\mathrm{M}$ & 1 & 1 & 1 & 1 \\
\hline 73 & $\mathrm{~F}$ & 1 & 1 & 1 & 0.7 \\
\hline 60 & M & 1 & 1 & 12 & 300 \\
\hline 73 & M & 1 & 1 & 2 & 4,432 \\
\hline 74 & $\mathrm{~F}$ & 1 & 1 & 1 & 14,400 \\
\hline 80 & M & 2 & 1 & 2 & 1,440 \\
\hline 78 & $M$ & 2 & 1 & 1 & 2 \\
\hline 61 & M & 4 & 1 & 1 & 180 \\
\hline 65 & $\mathrm{~F}$ & 4 & 1 & 2 & 1 \\
\hline 73 & $\mathrm{~F}$ & 4 & 1 & 4 & 300 \\
\hline 88 & M & 4 & 1 & 1 & 1,695 \\
\hline 72 & $M$ & 5 & 1 & 1 & 4,380 \\
\hline 96 & $\mathrm{~F}$ & 6 & 1 & 1 & 913 \\
\hline 88 & $\mathrm{~F}$ & 6 & 1 & 1 & 4,242 \\
\hline 62 & $M$ & 7 & 1 & 1 & 925 \\
\hline 83 & $\mathrm{~F}$ & 8 & 1 & 1 & 150 \\
\hline 70 & $M$ & 87 & 2 & 1 & 6 \\
\hline 64 & $\mathrm{M}$ & 90 & 2 & 1 & 439 \\
\hline 60 & $\mathrm{~F}$ & 91 & 2 & 3 & 4 \\
\hline 60 & $\mathrm{M}$ & 98 & 2 & 1 & 0.5 \\
\hline 75 & $\mathrm{~F}$ & 113 & 2 & 2 & 2 \\
\hline 72 & $\mathrm{~F}$ & 125 & 2 & 4 & 2,790 \\
\hline 74 & $\mathrm{~F}$ & 187 & 3 & 1 & 0.6 \\
\hline
\end{tabular}

During the first 6 months after randomization, two patients in the intervention arm had AF detection outside of the study intervention.

$A F$, atrial fibrillation; $E C G$, electrocardiography.

AF detection after 6 months

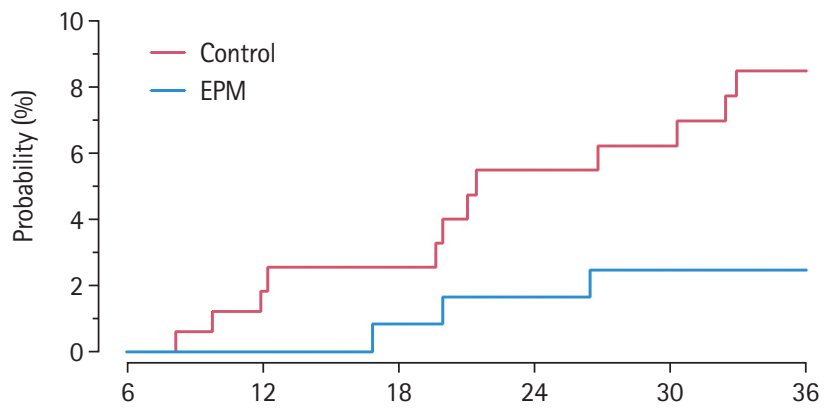

\begin{tabular}{rrrrrr} 
No. at risk & \multicolumn{5}{c}{ Months } \\
EPM 154 & 148 & 121 & 118 & 117 & 115 \\
Control 171 & 161 & 133 & 128 & 126 & 121
\end{tabular}

Supplementary Figure 1. Detection of AF conditional on no atrial fibrillation detection until 6 months. EPM, enhanced and prolonged monitoring. 